\title{
Design Optimization of CMOS Control Circuit for Integrated Photovoltaic Power Transfer
}

\author{
Takashi Tokuda, ${ }^{1 *}$ Takaaki Ishizu, ${ }^{1}$ Wuthibenjaphonchai Nattakarn, ${ }^{1}$ Makito Haruta, ${ }^{1}$ \\ Toshihiko Noda, ${ }^{1}$ Kiyotaka Sasagawa, ${ }^{1}$ Mohamad Sawan, ${ }^{2}$ and Jun Ohta ${ }^{1}$ \\ ${ }^{1}$ Graduate School of Materials Science, Nara Institute of Science and Technology, \\ 8916-5 Takayama-cho, Ikoma, Nara 630-0192, Japan \\ ${ }^{2}$ Department of Electrical Engineering, Polytechnique Montréal, \\ Montréal, QC H3T 1J4, Canada
}

(Received March 29, 2018; accepted September 18, 2018)

Keywords: CMOS, wireless, batteryless, photovoltaic power transfer, energy harvesting, implantable device, Internet of Things (IoT)

A CMOS control circuit for an integrated photovoltaic (PV) power transfer/harvesting platform is optimized. The proposed PV power transfer/harvesting platform is based on the concept of charging a small PV current in a capacitor and operating a target circuit intermittently. This architecture is suitable for various types of implantable or Internet of Things (IoT) devices. To realize the integration of PV cells on a CMOS chip, we need to improve the performance of the CMOS control circuit. We refined a self-powered voltage detector circuit in the CMOS control circuit to comply with small-current operation and improved the adjustability of the switching voltages. We describe a strategy and experimentally obtained results of the design optimization.

\section{Introduction}

Currently, various types of wireless power transfer technologies have been realized and utilized in both consumer and industry fields. Noncontact wireless power and data transfer for batteryless card terminals are commonly used in transportation and retailers. Wireless power transfer is also used to charge a battery in a cell phone and other gadgets. Most of the current wireless powering technologies are realized electromagnetically. Both power transmitter and receiver are equipped with $\mathrm{cm}$-sized coils, and the inductive coupling between two (primary and secondary) coils is used for power transfer. The electric power is once transformed into alternative current (AC) and rectified into direct current (DC) in power-receiving devices.

However, in terms of small devices such as implantable or distributed Internet of Things (IoT) nodes, there is no de facto standard of the wireless power transfer technology. For these types of applications, there are constraints in device dimensions and weight. In the field of implantable electronics and distributed IoT technologies, device dimensions less than $1 \mathrm{~cm}$ or even $1 \mathrm{~mm}$ are expected for the reduction in invasiveness (implantable electronics) or the aesthetics of items (IoT nodes). 
In the case of electromagnetic wireless transfer, ${ }^{(1-10)}$ the reduction in device size requires a reduction in the size of the secondary coil (diameter and number of turns). It causes a marked decrease in power transfer efficiency. It also requires a close placement of the primary and secondary coils. Consequently, for very small implantable or IoT devices, it is not reasonable to use the electromagnetic power transfer schemes.

The authors propose to use photovoltaic (PV) power ${ }^{(4,11-14)}$ transfer, thus, using solar cells for powering very small electronic devices. ${ }^{(15)}$ In general, the reduction in coil size for electromagnetic power transfer causes the reduction in both voltage and current. On the other hand, as a nature of solar cells, a reduction in the size of solar cells only leads to a reduction in generated photocurrent. The voltage from solar cells under sufficient illumination scarcely depends on the size of solar cells. We propose to take advantage of this intrinsic characteristic of solar cells. We use series-connected small solar cells as a power receiver with sufficient voltage and insufficient current.

Figure 1 shows the concept of the proposed PV power transfer scheme. ${ }^{(15)}$ Figure 2 shows a block diagram of the proposed PV power transfer/energy-harvesting platform. We integrate series-connected solar cells with a capacitor to charge the generated electric power. Adopting series-connected solar cells, we can obtain sufficient voltage for the operation of the target circuit without a voltage-boosting circuit such as a switching capacitor circuit. ${ }^{(12,13,16,17)} \mathrm{We}$ can wait to obtain a preset capacitor voltage and operate the target circuit for a short time. Thus, the target circuit should be designed to be compatible with the intermittent operation. This operating scheme is suitable for various types of implantable sensors and IoT micronodes with intermittent functionality. The proposed operating scheme has a nature of adaptiveness to the available power from the light. Operating frequency is automatically adjusted to fit the available energy.

On the basis of this concept, we realized an optically powered, batteryless optical ID transmission device. ${ }^{(15)}$ We used a set of $0.8 \times 0.9 \mathrm{~mm}^{2}$ discrete photodiodes as PV cells. We successfully obtained the optically powered operation of the device with an adaptiveness to

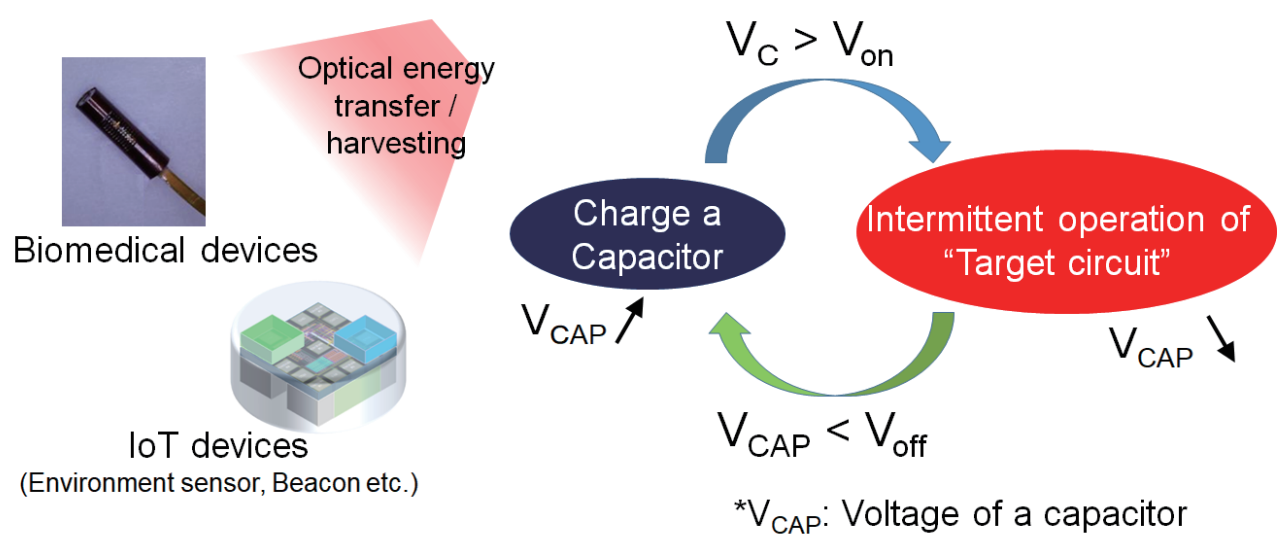

Fig. 1. (Color online) Concept of the CMOS-controlled PV power transfer scheme. 


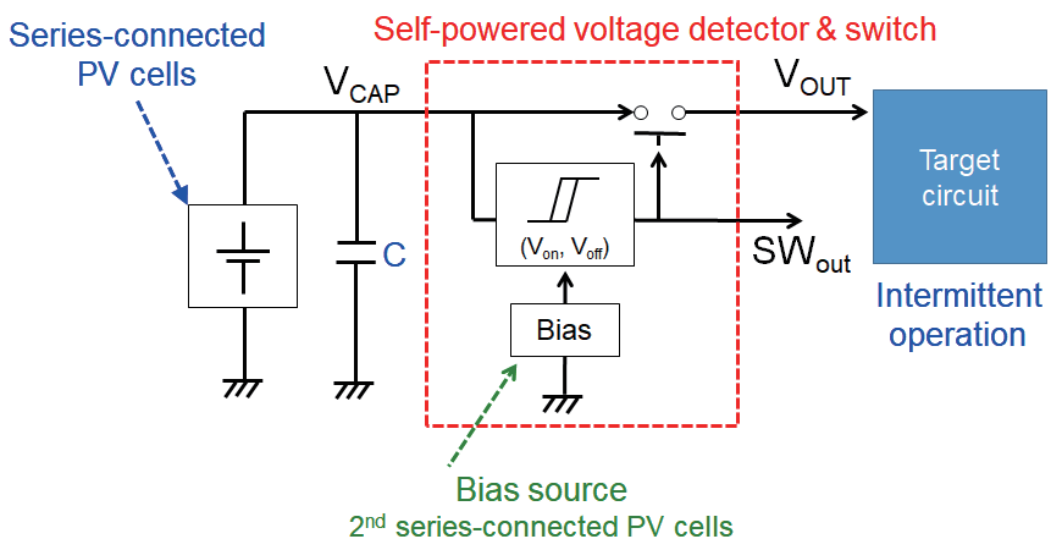

Fig. 2. (Color online) Block diagram of the CMOS-controlled PV power transfer platform.

the illumination. However, we found that relatively high intensity illumination is required to operate the device. The reduction in minimum operating intensity, i.e., photocurrent from series-connected PV cells, is required to realize mm-sized, batteryless microdevices.

In this work, we describe the optimization of the CMOS circuit design to reduce the minimum operating current. We also greatly improved the adjustability of the threshold voltages $V_{o n}$ and $V_{o f f}$, which are essential in the operation.

\section{Basic Design and Circuit Operation of the Proposed PV Power Transfer/ Energy-harvesting Platform}

The system shown in Fig. 2 consists of two sets of series-connected PV cells, a capacitor, a CMOS chip including a self-powered voltage detector, a powering switch, and a load circuit (target circuit). Off-chip loads such as light-emitting diodes (LEDs) can also be used. One of the series-connected PV cells is used for powering. The highest voltage node is connected to the capacitor, and photocurrent is accumulated in the capacitor. As the charge in the capacitor increases, the voltage of the capacitor, $V_{C A P}$, increases. Finally, we expect that $V_{C A P}$ can reach a maximum value that corresponds to the open-circuit voltage $\times$ number of series-connected PV cells. We can choose the appropriate number of PV cells to obtain a specific voltage range required to drive the load circuit. In this work, we adopted 10-series PV cells integrated on the CMOS chip. The typical open-circuit voltage of the integrated PV cells is $0.4 \mathrm{~V}$, and the maximum $V_{C A P}$ is approximately $4 \mathrm{~V}$.

$V_{C A P}$ is monitored by a self-powered voltage detector, and the connection from the capacitor to the load circuit is controlled by a CMOS switch. The self-powered voltage detector turns the CMOS switch on and off. To supply current to the load circuit for a specific duration, the voltage detector is designed to have a hysteresis. When $V_{C A P}$ reaches $V_{o n}$, the output of the voltage detector, $S W_{\text {out }}$, becomes high and the CMOS switch is closed to operate the load circuit. Once the voltage detector transits to high, the transition voltage from high to low is $V_{\text {off }}$, which is lower than $V_{o n}$. The operation of the load circuit causes a discharge of the capacitor and a 
decrease in $V_{C A P}$. The CMOS switch is kept on before $V_{C A P}$ goes down to $V_{\text {off. }}$. $V_{\text {on }}$ and $V_{\text {off }}$ as well as capacitance, should be designed to match with the voltage range and current required for the operation of the load circuit. For example, in the case of driving a blue LED as a load, $V_{\text {on }}=3.5 \mathrm{~V}$ and $V_{\text {off }}=2.5 \mathrm{~V}$ can be adopted as threshold voltages. The capacitance should be chosen to match with an expected operation duration of the LED. The self-powered voltage detector itself is driven by the voltage that the circuit monitors. Currently, the voltage detector needs some additional constant bias voltages independent of the powering voltage. We used the second set of series-connected PV cells to provide the bias voltages for the self-powered voltage detector. We take bias voltages at intermediate nodes of the second series-connected PV cells. These bias voltages are connected only to gates of MOS transistors in the circuit. Since no continuous current flows into the gates of MOS transistors, as soon as the PV cells are illuminated, all the intermediate nodes of the second series-connected PV cells rapidly reach a quasi-terminal state in which we obtain an open-circuit voltage from each cell. As a drawback of this simple approach, there is a constraint that we can use only quantized voltages and thus a simple multiplication of the open-circuit voltage of the on-chip PV cell $(\sim 0.4 \mathrm{~V})$. It should be noted also that the voltages delivered from the series-connected PV cells slightly depend on the illumination.

Figure 3 shows two versions of the self-powered voltage detector circuit. Figure 3(a) shows the original circuit configuration employed in the previous work. ${ }^{(15)}$ Figure 3(b) shows the improved circuit described in this work. We introduced two additional MOS transistors $\left(\mathrm{M}_{\mathrm{n} 4}\right.$ and $\left.\mathrm{M}_{\mathrm{n} 5}\right)$ and one additional bias input $\left(V_{b p 2}\right)$. The functions of the additional elements are described in the next section.

The basic self-powered voltage detector circuit [Fig. 3(a)] consists of series-connected three stages of digital components. The second and third stages are conventional inverters. The main features of the circuit, i.e., self-powered voltage detection and hysteresis, are realized by the first-stage switch. Different from the conventional inverter, the gates of the PMOS $\left(\mathrm{M}_{\mathrm{p} 1}\right)$ and NMOS $\left(\mathrm{M}_{\mathrm{n} 1}\right)$ transistors are biased with fixed voltages, which come from the second set of series-connected PV cells shown in Fig. 2. The operation of the voltage detector shown in Fig. $3(\mathrm{a})$ is as follows.

In the initial stage of the operation, when the first (powering) and second (biasing) seriesconnected PV cells are illuminated, both sets of PV cells generate photocurrent. Since the powering PV cells are connected to the capacitor, the photocurrent is charged to the capacitor and $V_{C A P}$ increases gradually. On the other hand, in terms of the biasing PV cells, only some nodes are connected to MOS transistors in the CMOS circuit, and there is no current-draining path. The biasing PV cells rapidly get to a stable state and provide bias voltages to the CMOS circuit.

The initial switching behavior is determined by the two MOS transistors $M_{n 1}$ and $M_{p 1}$. The source and gate terminals of $\mathrm{M}_{\mathrm{n} 1}$ are at fixed voltages, thus the source-gate voltage $V_{G S}$ is equal to $V_{b n} . \mathrm{M}_{\mathrm{n} 1}$ gives some conductivity for the whole range of drain voltage $V_{1}$, the output voltage of the first-stage inverter. On the other hand, the source voltage of $\mathrm{M}_{\mathrm{p} 1}$ is $V_{\text {in }}=V_{C A P}$ and the gate voltage is $V_{b p}$. Therefore, the $V_{G S}$ for $\mathrm{M}_{\mathrm{p} 1}$ depends on $V_{C A P}$. In the start-up stage of the PV power supply, $V_{C A P}$ is low and $V_{G S}$ (we use $V_{G S}=V_{S}-V_{G}$ for PMOS) is negative. Because of 


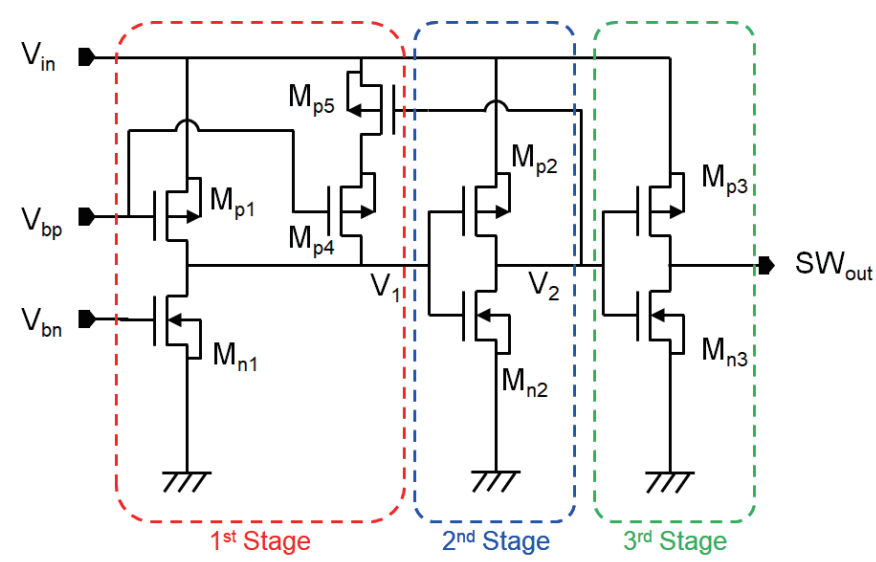

(a)

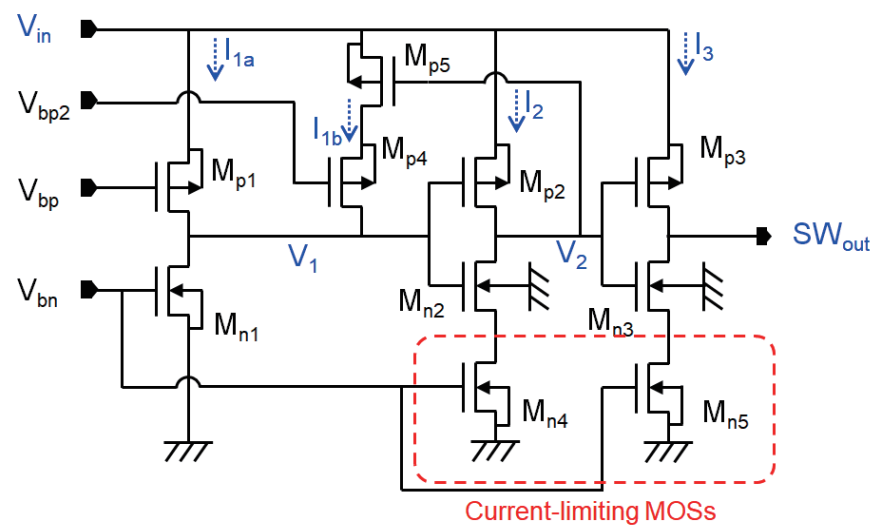

(b)

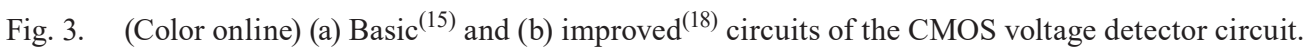

the negative $V_{G S}, \mathrm{M}_{\mathrm{p} 1}$ is turned off. The conductive $\mathrm{M}_{\mathrm{n} 1}$ and the resistive $\mathrm{M}_{\mathrm{p} 1}$ pull down $V_{1}$ to $0 \mathrm{~V}$, and it gives $V_{2}=V_{\text {in }}$ and $S W_{\text {out }}=0 \mathrm{~V}$. The additional current pathway that consisted of two PMOSs, $\mathrm{M}_{\mathrm{p} 4}$ and $\mathrm{M}_{\mathrm{p} 5}$, is not activated because $V_{G S}=0$ for $\mathrm{M}_{\mathrm{p} 5}$.

When PV cells are continuously illuminated, the photocurrent provided by the powering PV cells flows into the capacitor and $V_{C A P}$ gradually increases. The $V_{G S}$ of $\mathrm{M}_{\mathrm{p} 1}$ will turn positive and finally reach the threshold voltage of $\mathrm{M}_{\mathrm{p} 1}$ (typically $0.4-0.6 \mathrm{~V}$ for a $0.35 \mu \mathrm{m}$ process that we use in this work) and higher. When the conductivity of $\mathrm{M}_{\mathrm{p} 1}$ becomes larger than that of $\mathrm{M}_{\mathrm{n} 1}, V_{1}$ becomes high $\left(=V_{i n}\right)$. It causes transitions of the second- and third-stage inverters and gives $V_{2}=0 \mathrm{~V}$ and $S W_{\text {out }}=V_{\text {in }}$. We mention the $V_{\text {in }}$ level for this transition as the "turnon threshold voltage $V_{\text {on }}$ ". $S W_{\text {out }}$ is the output of this voltage detector circuit that controls the connection between the capacitor (power source part) and the load (power-consuming part). In the configuration shown in Fig. 2, the CMOS switch between the capacitor and the load is connected and the electric power is delivered from the capacitor to the target load circuit. 
At the same time, $V_{2}=0$ turns on $\mathrm{M}_{\mathrm{p} 5}$ and activates the additional pathway in the first-stage switch. The additional current path configured by $\mathrm{M}_{\mathrm{p} 4}$ and $\mathrm{M}_{\mathrm{p} 5}$ gives additional conductivity between the $V_{1}$ node and $V_{i n}$, which helps to keep $V_{1}$ high $\left(=V_{i n}\right)$.

In most cases, the photocurrent available from the powering PV cells is smaller than the current that flows into the load circuit, and it causes a discharge of the capacitor and a decrease in $V_{C A P}\left(=V_{i n}\right)$. However, since the $\mathrm{M}_{\mathrm{p} 4}-\mathrm{M}_{\mathrm{p} 5}$ current path is activated, the first-stage switch does not turn off at $V_{o n}$. The first-stage switch turns off at $V_{\text {off, }}$, which is lower than $V_{o n}$.

Figure 4 shows an experimentally obtained cyclic operation between the charging phase and the discharging phase. $V_{C A P}\left(=V_{i n}\right)$ repeatedly goes up to $V_{o n}$ and down to $V_{\text {off }}$ during the operation. The charging time depends on the photocurrent from the powering PV cells, thus illuminating the cells. Figure 5 shows the start-up charging time as a function of the charging current. The start-up charging time is the time to charge the capacitor from $V_{C A P}=0$ to $V_{\text {on }}$. The start-up charging time in Fig. 5 was measured in a bench-top evaluation using a current source to charge the capacitor and voltage sources to provide $V_{b n}$ and $V_{b p}$. We obtained $2 \mu \mathrm{A}$ as the minimum charging current for the automatic charge and discharge operation. The voltage detector shown in Fig. 3(a) did not correctly work with a current smaller than $2 \mu \mathrm{A}$.

The hysteresis of the first-stage switch, thus, the mismatch between $V_{o n}-V_{o f f}$, determines what amount of charge will be provided from the capacitor to the load circuit. The capacitance $C$ and the impedance of the load circuit also affect the operation duration of the load circuit.

Figure 6 shows the threshold voltages (a) $V_{o n}$ and (b) $V_{o f f}$, and (c) hysteresis $V_{o n}-V_{o f f}$ as functions of $V_{b n}$ and $V_{b p}$. As shown in Figs. 6(a) and 6(b), the threshold voltages depend on both $V_{b n}$ and $V_{b p}$. We can adjust $V_{o n}$ and $V_{o f f}$ by changing the bias conditions. On the other hand, as shown in Fig. 6(c), the hysteresis $V_{o n}-V_{o f f}$ is almost independent of $V_{b p}$. In fact, although $V_{o n}-V_{\text {off }}$ slightly depends on $V_{b n}$, it is difficult to change $V_{b n}$ only to adjust the hysteresis. An increase in $V_{b n}$ causes a larger continuous current during the operation and a poor circuit performance.

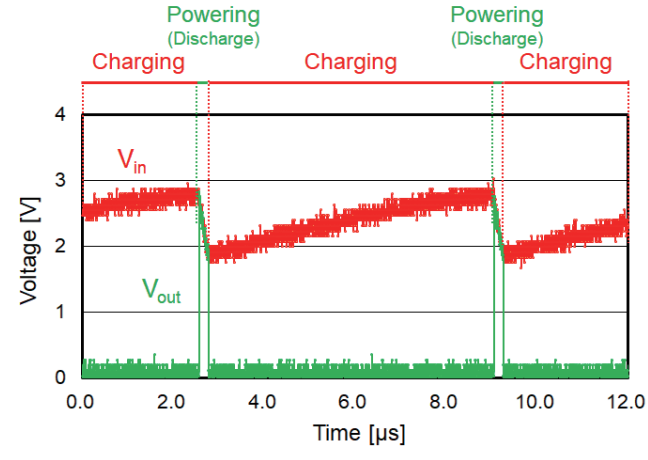

Fig. 4. (Color online) Experimental trace showing the charge and operation cycle.

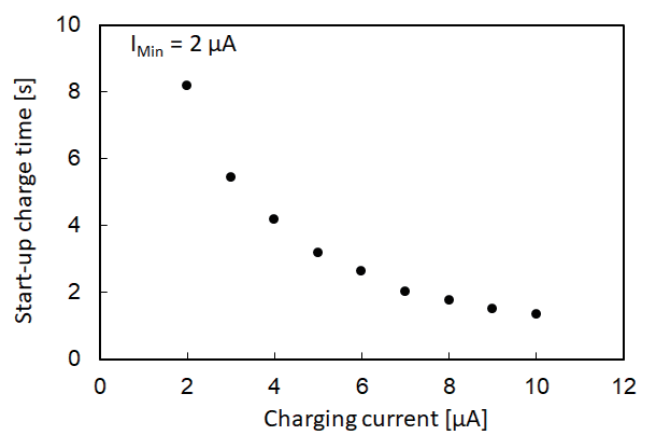

Fig. 5. Start-up charging time as a function of charging current. 


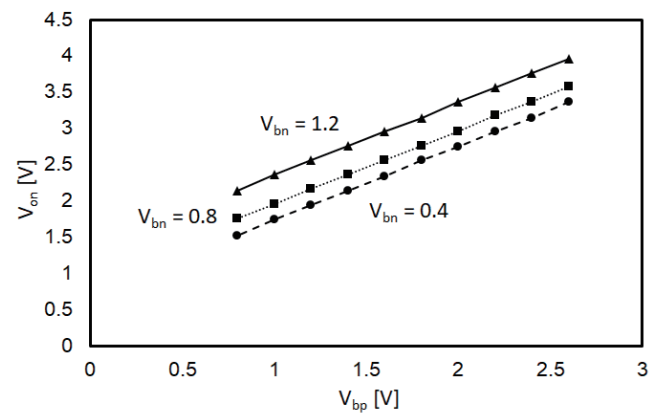

(a)

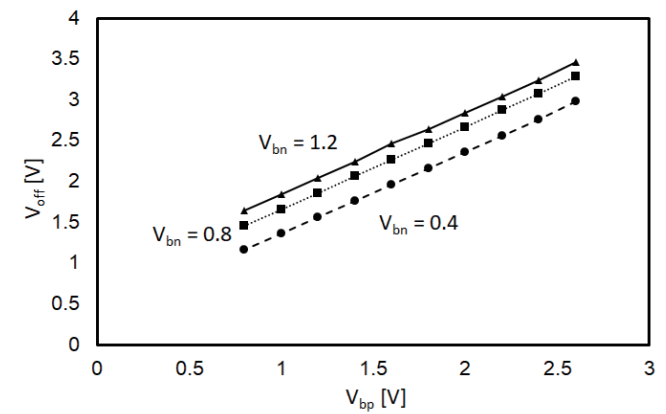

(b)

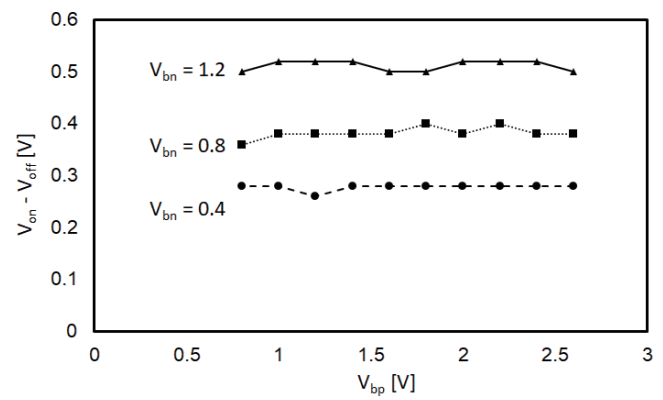

(c)

Fig. 6. Threshold voltages (a) $V_{o n}$ and (b) $V_{o f f}$, and (c) hysteresis $V_{o n}-V_{o f f}$ as functions of $V_{o n}$ and $V_{b p}$.

\section{Design Optimization of the Voltage Detector Circuit}

\subsection{Design issues to be resolved}

As presented in the previous section, although the CMOS-controlled optical power receiver platform shown in Fig. 3(a) works as we expected, there are two issues to be improved. The first issue is that the minimum charging current to obtain the expected operation is as large as $2 \mu \mathrm{A}$. In a previous work, ${ }^{(15)}$ we used externally configured $0.8 \times 0.9 \mathrm{~mm}^{2}$ Si photodiodes as the PV cells. A photocurrent on the order of $\mu \mathrm{A}$ or even larger is available from the Si PV cell chip, and we can drive an optical ID transmission circuit with the proposed PV optical power transfer scheme. As next-generation optically powered, batteryless microdevices, we plan to use on-chip photodiode structures as integrated PV cells. The photocurrent available from the integrated PV cell is expected to be significantly smaller than that from the above-mentioned $\mathrm{Si}$ PV cell. We need to improve the circuit to reduce the minimum operating current.

The other issue is that we cannot adjust the hysteresis $V_{o n}-V_{o f f}$. Once the circuit is designed, $V_{o n}-V_{\text {off }}$ scarcely depends on the bias voltages $V_{b n}$ and $V_{b p}$. In other words, we cannot set $V_{o n}$ and $V_{\text {off }}$ independently. To apply this platform to various target functions, we need to realize the mutually independent adjustment of $V_{o n}$ and $V_{\text {off }}$ by changing the bias voltages. In this section, circuit modifications to overcome these two issues are described. 


\subsection{Introduction of current-limiting MOS transistors and reduction in minimum operating current}

In general, a CMOS logic circuit such as a CMOS inverter employed in the current voltage detector does not require a continuous current flow in the steady state. The current flows only in transition of the logic states. Some circuit simulations revealed that the instantaneous current flow during the transition causes incomplete turning-on and the circuit stacks in a metastable state. With a small input current, as long as the CMOS voltage detector is in the off-state and the CMOS switch is opened, the photocurrent can charge the capacitor. This is because all the digital circuits are in the steady state and the total current for the CMOS circuit is very low. However, once $V_{C A P}$ reaches $V_{\text {on }}$, the CMOS voltage detector starts to transit from the off-state to the on-state. The digital circuits in the voltage detector drain all the photocurrent generated in the powering PV cells. Under a low-current condition, the voltage detector circuit cannot complete the transition from the off-state to the on-state and the CMOS switch between the capacitor and the load is still opened. $V_{C A P}$ can neither go up to complete the transition nor drop down to restart the charging, because the current does not flow into the load circuit. This is the reason why the minimum operating current is as large as $2 \mu \mathrm{A}$ for the circuit shown in Fig. 3(a).

The straightforward solution to overcome this issue is to limit the transition current of the CMOS circuits. In the revised circuit design [Fig. 3(b)], we introduced additional NMOS transistors (current-limiting NMOS transistors) in each of the inverter structures in Fig. 3(a). In the circuit shown in Fig. 3(b), all NMOS transistors were designed as $W / L=1.0 \mu \mathrm{m} / 0.5 \mu \mathrm{m}$, and all PMOS transistors were designed as $W / L=2.0 \mu \mathrm{m} / 0.5 \mu \mathrm{m}$.

To understand the effects of current limitation, we performed simulations for a slightly modified circuit from Fig. 3(b). We applied a separate bias voltage $V_{b n 2}$ to gates of the currentlimiting NMOS transistors $\left(\mathrm{M}_{\mathrm{n} 4}\right.$ and $\left.\mathrm{M}_{\mathrm{n} 5}\right)$. This means that $V_{b n}$ is applied only to the gate of the $\mathrm{M}_{\mathrm{n} 1}$ transistor. Figure 7 shows the simulated transient traces indicating voltages and currents on the circuit. The traces are plotted around the switching operation. In Fig. 7(a), $V_{b n 2}$ is set as $3.0 \mathrm{~V}$, with which current is not limited. Once $V_{\text {in }}$ reaches $V_{\text {on }}$ (around $2.8 \mathrm{~V}$ in this case), the current flow through the second-stage inverter $\left(\mathrm{I}_{2}\right)$ increases and most of the photocurrent $(1 \mu \mathrm{A})$ is drained through the second-stage inverter. This prevents the circuit from the expected turn-on operation and no pulse is generated. On the other hand, with $V_{b n 2}=0.3$ $\mathrm{V}$, in the switching operation, $\mathrm{I}_{2}$ only shows a short spike with a peak current of approximately $150 \mathrm{nA}$, and the circuit correctly generates a pulse. With the gate voltage of $0.3 \mathrm{~V}$, the currentlimiting NMOS transistors successfully limit the transition current of the inverter circuits.

From the viewpoint of voltage availability from the serial-connected PV cells for biasing, a single-PV cell level $(\sim 0.4 \mathrm{~V})$ was chosen as the bias voltage $V_{b n}$ for the current-limiting NMOS transistors. Simulations suggest that the circuit works with an operating current of $200 \mathrm{nA}$ from the powering PV cells.

\subsection{Introduction of additional bias voltage for independent setting of $V_{o n}$ and $V_{o f f}$}

In the previous design [Fig. 3(a)], the hysteresis of the first-stage switching circuit is realized with the additional current path that is activated only in the on-state. We applied the same bias 


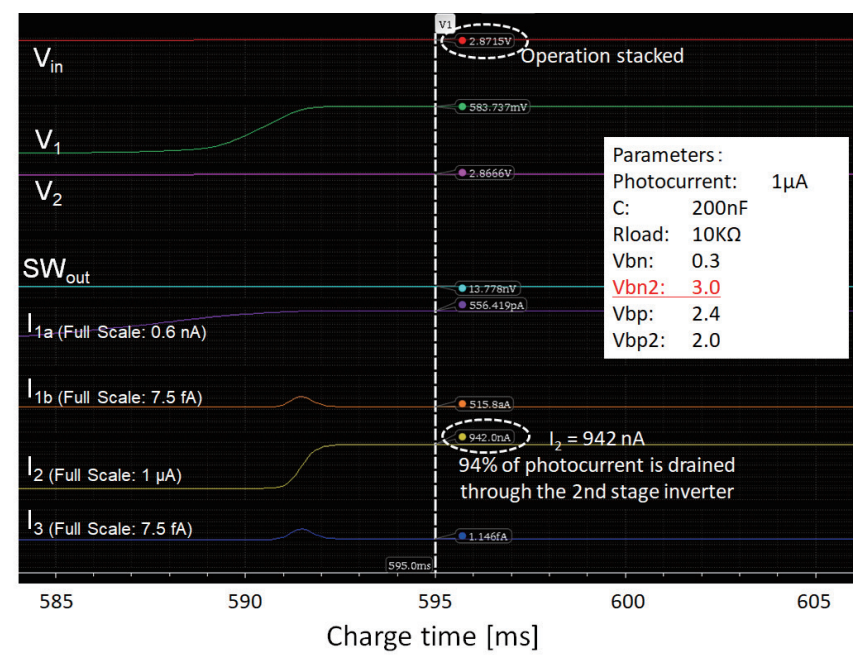

(a)

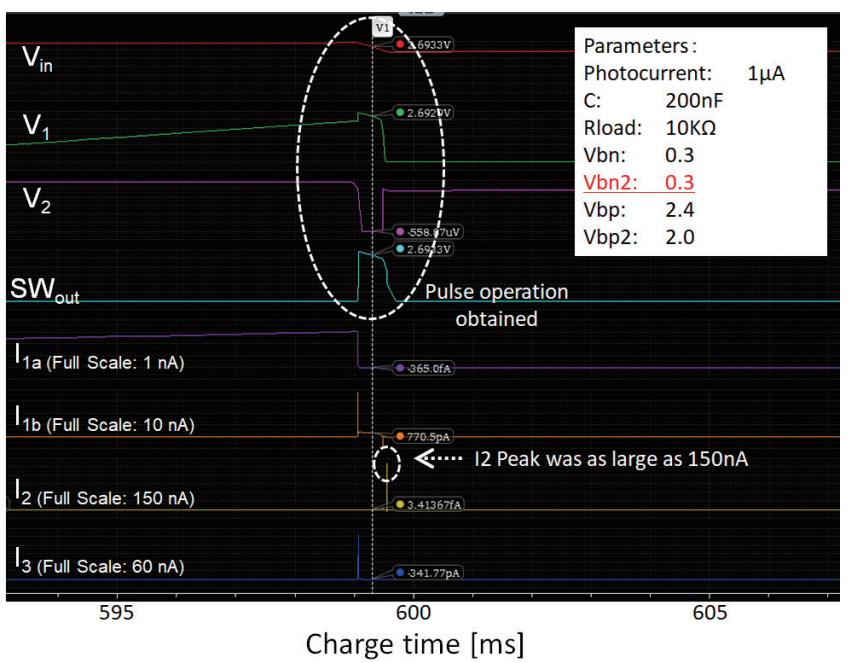

(b)

Fig. 7. (Color online) Simulated circuit operation shown in Fig. 3(b): (a) without and (b) with current-limiting condition.

voltage $V_{b p}$ to both PMOS transistors $\left(\mathrm{M}_{\mathrm{p} 1}\right.$ and $\left.\mathrm{M}_{\mathrm{p} 4}\right)$. Simulations suggested that separating the gate voltages of these two PMOS transistors makes it possible to change $V_{\text {on }}$ and $V_{\text {off }}$ independently. Thus, we introduced the second PMOS bias $V_{b p 2}$ only for $\mathrm{M}_{\mathrm{p} 4}$, as shown in Fig. $3(b)$.

\section{Chip Implementation and Experimental Evaluation}

\subsection{CMOS chip design}

On the basis of the optimization described in the previous section, we designed two CMOS chips for evaluation and demonstration. We used a $0.35 \mu \mathrm{m}$ 2-poly, 4-metal standard CMOS 
process. Figure 8 shows layouts of the CMOS chips. Table 1 shows the specifications of the chips.

Chip-A is a voltage-monitoring circuit [see Fig. 3(b)] for evaluating the reduction in operating current and improving the adjustability of the threshold voltages. Chip-B is the integrated optical power receiver/energy-harvesting chip. We integrated the voltage detector and the CMOS switch with integrated PV cells. We used an N-well/P-sub photodiode structure commonly available in standard CMOS processes as the on-chip PV cell. Two sets of seriesconnected on-chip PV cells were integrated on chip-B. The first set is composed of 10-seriesconnected PV cells with a size of $270 \times 270 \mu \mathrm{m}^{2}$ for powering. The photocurrent from the anode terminal of the 10th PV cell is accumulated in a capacitor. Since approximately 0.4 $\mathrm{V}$ is available from a single PV cell, we expect that more than $4 \mathrm{~V}$ will be available from the powering PV cells. The second set is composed of 7-series-connected PV cells with a size of $120 \times 170 \mu \mathrm{m}^{2}$. We call such PV cells as the biasing PV cells. We took the bias voltage from the intermediate nodes of the biasing PV cells. We took approximately $0.4 \mathrm{~V}$ from the $\mathrm{PV} \times$ 1 node for $V_{b n}, 2.0 \mathrm{~V}$ from the PV $\times 5$ node for $V_{b p 2}$, and $2.8 \mathrm{~V}$ from the highest (7th) node for $V_{b p}$. These bias connections were chosen on the basis of simulations and data obtained experimentally with chip-A, which will be described in Sects. 4.2 and 4.3.

The optimized circuit shown in Fig. 3(b) was characterized with Chip-A. All the bias levels $\left(V_{b n}, V_{b p}\right.$, and $\left.V_{b p 2}\right)$ were supplied from a DC voltage source. Figure 9 shows setups for evaluation. To evaluate the operating current described in Sect. 4.2, a 1-10 $\mu \mathrm{F}$ capacitor was connected between $V_{\text {in }}$ and GND and a resistor load was connected between $V_{\text {out }}$ and GND. We used a current source connected between $V_{i n}\left(V_{C A P}\right)$ and GND to charge the capacitor. On the other hand, to evaluate the threshold voltages (Sect. 4.3), the experiment was performed without the capacitor and resistor load. We simply applied a triangular voltage wave from a function generator to identify the switching threshold voltages $V_{\text {on }}$ and $V_{\text {off }}$.

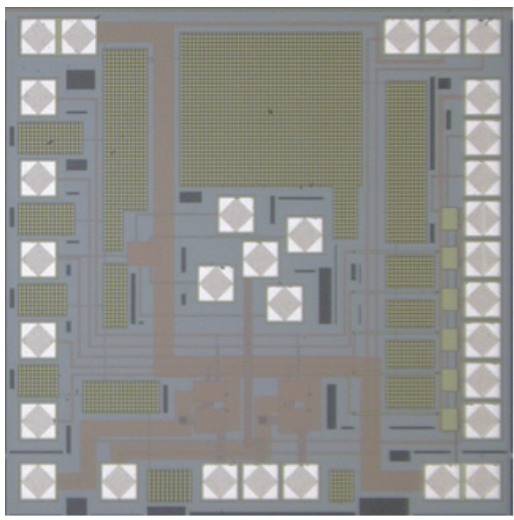

(a)

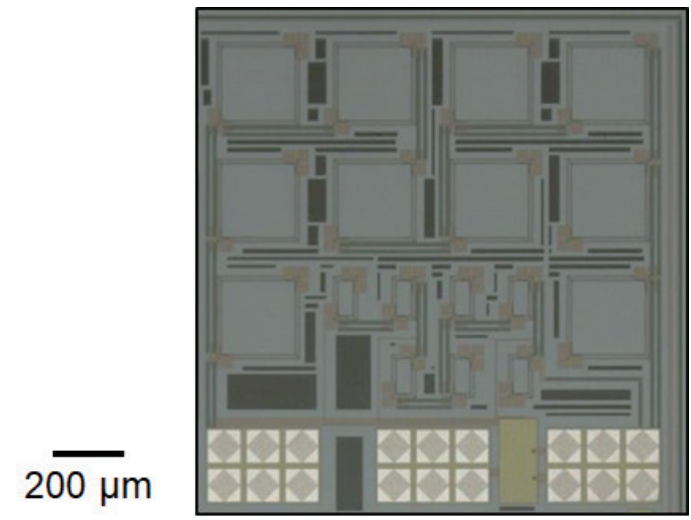

(b)

Fig. 8. (Color online) Layouts of the CMOS chips for (a) Chip-A, evaluation of the improved voltage monitoring circuit and (b) Chip-B, functional demonstration. 
Table 1

Specifications of the CMOS chips.

\begin{tabular}{lcc}
\hline & Chip-A & Chip-B \\
\hline Layout & Fig. 8(a) & Fig. 8(b) \\
Process & $0.35 \mu \mathrm{m}$, 2-poly, 4-metal standard CMOS process \\
Standard voltage & \multicolumn{2}{c}{$3.3 \mathrm{~V}$} \\
Target operating voltage & $2.5-3.5 \mathrm{~V}$ \\
Purpose & Circuit evaluation & Demonstration \\
Chip size & $1.25 \times 1.25 \mathrm{~mm}^{2}$ & (LED drive) \\
Voltage detector circuit & 1 & $1.25 \times 1.25 \mathrm{~mm}^{2}$ \\
Integrated on-chip PV cells & 0 & 1 \\
$V_{\text {in }}$ and bias voltages & Externally provided & Internally provided \\
\hline
\end{tabular}

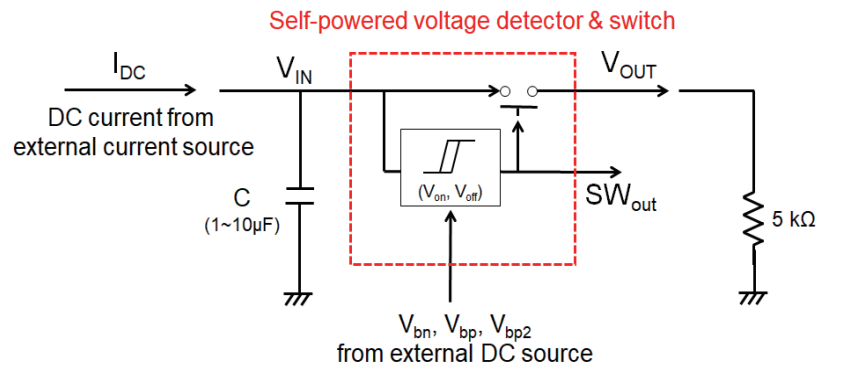

(a)

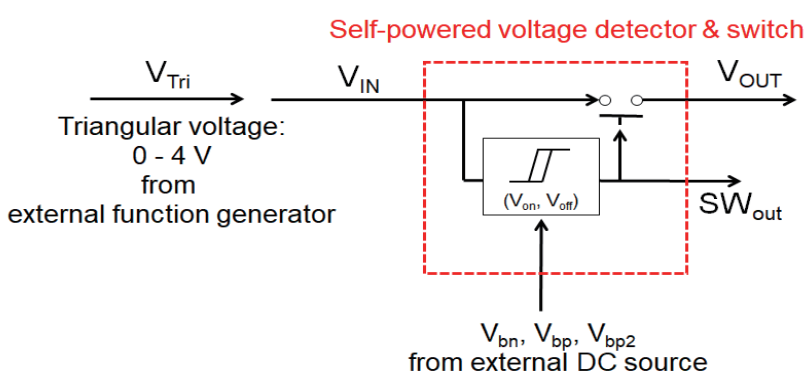

(b)

Fig. 9. (Color online) Experimental setups for measurements of (a) minimum operating current and (b) $V_{o n}$ and $V_{\text {off. }}$

\subsection{Small current operation of the CMOS energy-harvesting switch}

With the setup shown in Fig. 9(a), we evaluated the minimum operating current. We set $V_{b p}=2.8 \mathrm{~V}$ and $V_{b p 2}=2.0 \mathrm{~V}$, and changed $V_{b n}$ from 0 to $1.2 \mathrm{~V}$. We measured the minimum operating current at which automatic switching between $V_{\text {on }}$ and $V_{\text {off }}$ is available. Figure 10 shows the dependence of the minimum operating current on $V_{b n}$. The minimum operating current strongly depends on $V_{b n}$. The smaller the $V_{b n}$, the larger the current-limiting effect. We obtained an operating current of $370 \mathrm{nA}$ with $V_{b n}=0.4 \mathrm{~V}$. This operating current is approximately one order smaller than $2 \mu \mathrm{A}$, which was obtained with the previous circuit design [Fig. 3(b)]. ${ }^{(15)}$ This results shows that the introduction of the NMOS for the current-limiting is effective in realizing the low-current operation. The minimum operation current is determined mainly by the current flowing either of the current-limiting NMOS transistors $\left(M_{n 4}\right.$ or $\left.M_{n 5}\right)$. These transistors are in the subthreshold region throughout the operation. We consider that mismatch between the minimum operating current obtained in the simulation (200 nA) and that in the experiments $(370 \mathrm{nA})$ is caused by process fluctuation in the CMOS technology. 


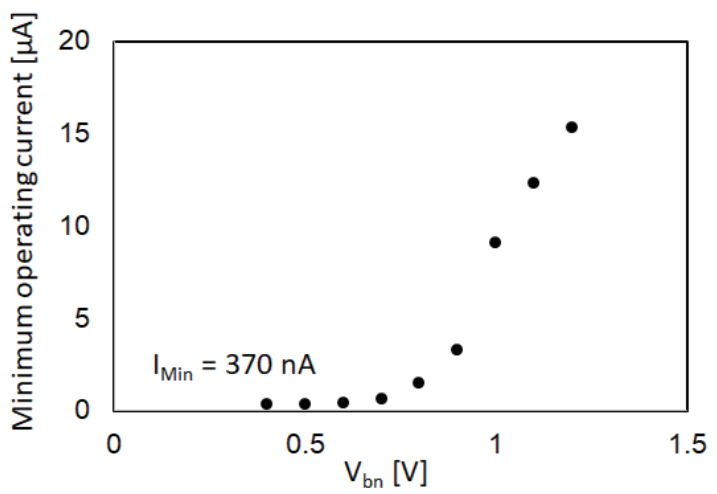

Fig. 10. Minimum operating current as a function of $V_{b n}$.

\subsection{Adjustability of threshold voltages}

Figure 11 shows $V_{o n}$ and $V_{\text {off }}$ as functions of (a) $V_{b p}$ and (b) $V_{b p 2}$. For Fig. 11(a), $V_{b p 2}$ was fixed at $2.0 \mathrm{~V}$. For Fig. 11(b), $V_{b p}$ was fixed at $2.8 \mathrm{~V}$. To understand the characteristic of the circuit, we need to add a condition. In a real operation, $V_{b p}$ should be higher than $V_{b p 2}$ based on the circuit architecture. With this additional condition, the bias voltage ranges of $V_{b p}>2.0$ $\mathrm{V}$ in Fig. 11(a) and $V_{b p 2}<2.8 \mathrm{~V}$ for Fig. 11(b) should be discussed. In Fig. 11(a), $V_{o n}$ linearly increases with $V_{b p}$ in the whole voltage range. On the other hand, when we focus on the voltage range with $V_{b p}>2.0 \mathrm{~V}, V_{\text {off }}$ does not depend on $V_{b p}$. This means that only $V_{o n}$ can be adjusted by changing $V_{b p}$, and $V_{o f f}$ is independent of $V_{b p}$. On the other hand, in Fig. 11(b), $V_{o n}$ does not depend on $V_{b p 2}$, and $V_{o f f}$ linearly increases with $V_{b p 2}$. This result suggests that $V_{o f f}$ is solely adjustable by changing $V_{b p 2}$ and that $V_{o n}$ is independent of $V_{b p 2}$. We can conclude that the introduction of $V_{b p 2}$ to bias $\mathrm{M}_{\mathrm{p} 4}$ leads to the separated adjustabilities of $V_{\text {on }}$ and $V_{\text {off. }}$.

A small fluctuation in $V_{\text {on }}$ up to approximately $0.2 \mathrm{~V}$ in Fig. 11(b) was observed. From a viewpoint of the concept of the system, this level of fluctuation causes no problem. One of the core advantages of the present wireless optical powering architecture is its adaptability to a wide illumination intensity range. The powering pulse is a simple discharging pulse taken from a capacitor and not regulated. Furthermore, as mentioned in Sect. 2, we obtain bias voltages from the second set of PV cells whose voltages slightly depend on illumination. We accept fluctuations in pulse parameters, such as initial $\left(\sim V_{\text {on }}\right)$ and final $\left(\sim V_{\text {off }}\right)$ voltages, pulse duration, and voltage transient of the powering pulse. $V_{\text {on }}$ and $V_{\text {off }}$ should be configured within an operating voltage range of the target circuit. At the same time, the target circuit should be sufficiently voltage-tolerant to work with the nonuniform, unstable powering pulse. We design systems with typical voltage ranges larger than $1 \mathrm{~V}^{(14,15,18)}$ and the fluctuation of $0.2 \mathrm{~V}$ is acceptable for our applications.

\subsection{Application demonstration: implantable optogenetic stimulator}

After the characterization with the voltage switching circuit, we designed the CMOS powerreceiving chip shown in Fig. $8\left(\right.$ b). ${ }^{(18)}$ To use chip-B, we need to separate the on-chip PV cells 


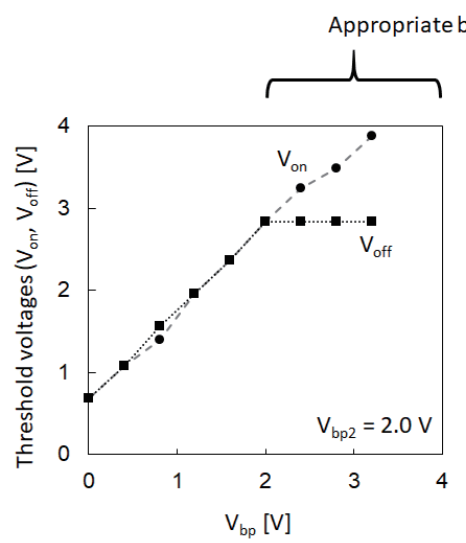

(a)

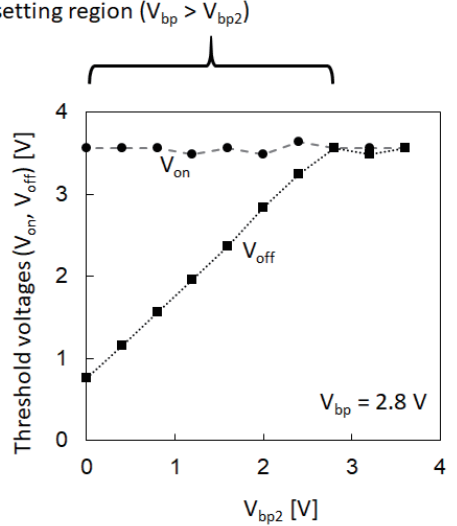

(b)

Fig. 11. $V_{o n}$ and $V_{o f f}$ as functions of (a) $V_{b p}$ and (b) $V_{b p 2} . V_{b p 2}$ was fixed at $2.0 \mathrm{~V}$ for (a) and $V_{b p}$ at $2.8 \mathrm{~V}$ for (b).

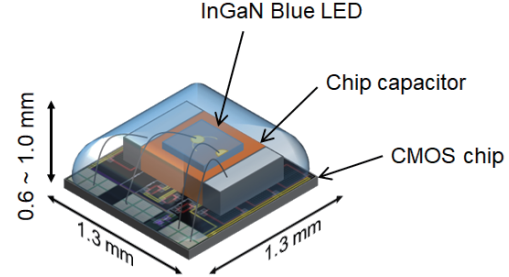

(a)

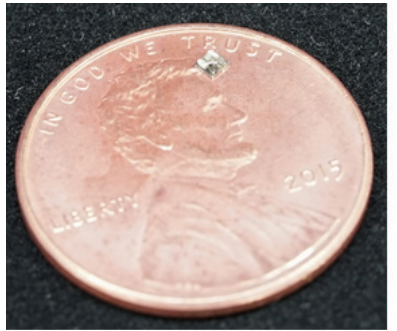

(b)

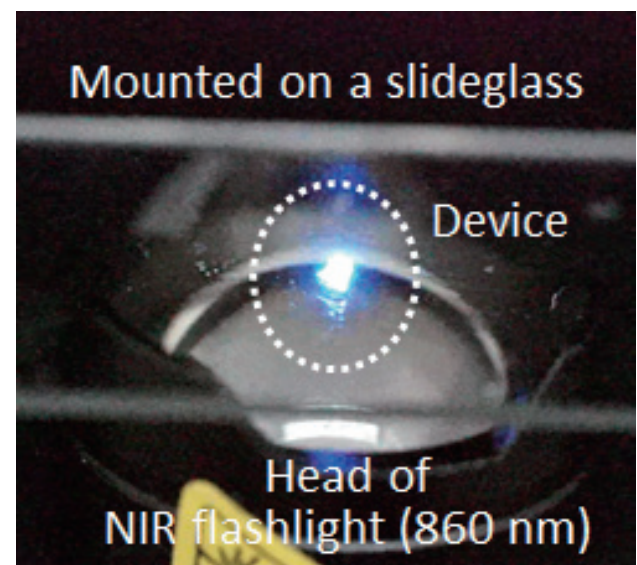

(c)

Fig. 12. (Color online) (a) Structure and (b) outlook of a batteryless implantable optogenetic neural stimulator based on the proposed CMOS-based optical power transfer platform. (c) A photograph of the device under operation. ${ }^{(18)}$

using the Bosch process. We described the process and integration of the chip with a capacitor and an InGaN blue LED in Ref. 18. Figure 12 shows an implantable optogenetic stimulator with the CMOS integrated PV power-receiving chip. ${ }^{(18)}$ The volume of the device is approximately $1 \mathrm{~mm}^{3}$ and the weight of the device is as small as $2.3 \mathrm{mg}$. Figure 12(c) shows a photograph of the device under operation. The device was placed on a slide glass and illuminated by a NIR flash light with a peak emission wavelength of $860 \mathrm{~nm}$. As shown in Fig. 12(c), we successfully drove a blue LED. The peak illumination intensity was higher than $10 \mathrm{~mW} / \mathrm{mm}^{2}$ and the pulse 
duration is longer than $1 \mathrm{~ms}$. Detailed characteristics are presented in Ref. 18. We expect to apply this device to a research field of optogenetics.

\section{Conclusions}

In this work, we optimized a CMOS circuit design for an optical power transfer/energyharvesting platform. The proposed optical power transfer/energy-harvesting platform is designed with a concept of a cyclic operation between the accumulation of PV current in a capacitor and the intermittent operation of the target circuit. The proposed technology is suitable for various types of implantable bioelectronics and IoT micronodes.

On the basis of our previous work, we refined the CMOS circuit to obtain a reduced operating current and flexible adjustability of the switching voltages. We introduced currentlimiting NMOS transistors for the reduction in operation current and an additional bias voltage for separate adjustabilities of the turn-on and turn-off voltages of the voltage detector.

We implemented the refined CMOS circuit in a real chip and obtained successful results for the two issues. Finally, we briefly mentioned the implantable optogenetic stimulator, which is realized with the CMOS circuit optimized in this work.

\section{Acknowledgments}

This work was supported in part by the Japan Science and Technology Agency under PRESTO \#JPMJPR1689, and by the Japan Society for the Promotion of Science under KAKENHI \#15KK0209 and \#17H02222. The CMOS chips were designed with the support of the VLSI Design and Education Centre (VDEC), the University of Tokyo, in collaboration with the Cadence Corporation and the Mentor Graphics Corporation.

\section{References}

1 H. M. Lee, H. Park, and M. Ghovanloo: IEEE J. Solid-State Circuits 48 (2013) 2203. https://doi.org/10.1109/ JSSC.2013.2266862

2 A. J. Yeh, J. S. Ho, Y. Tanabe, E. Neofytou, R. E. Beygui, and A. S. Y. Poon: Appl. Phys. Lett. 103 (2013) 163701. https://doi.org/10.1063/1.4825272

3 K. L. Montgomery, A. J. Yeh, J. S. Ho, V. Tsao, S. Mohan Iyer, L. Grosenick, E. A. Ferenczi, Y. Tanabe, K. Deisseroth, S. L. Delp, and A. S. Y. Poon: Nat. Methods 12 (2015) 969. https://doi.org/10.1038/nmeth.3536

4 S. Il Park, G. Shin, A. Banks, J. G. McCall, E. R. Siuda, M. J. Schmidt, H. U. Chung, K. N. Noh, J. G.-H. Mun, J. Rhodes, M. R. Bruchas, and J. A. Rogers: J. Neural Eng. 12 (2015) 056002. https://doi.org/10.1088/17412560/12/5/056002

5 D. Ahn and M. Ghovanloo: IEEE Trans. Biomed. Circuits Syst. 10 (2016) 125. https://doi.org/10.1109/ TBCAS.2014.2370794

6 T.-i. Kim, J. G. McCall, Y. H. Jung, X. Huang, E. R. Siuda, Y. Li, J. Song, Y. M. Song, H. A. Pao, R.-H. Kim, C. Lu, S. D. Lee, I.-S. Song, G. Shin, R. Al-Hasani, S. Kim, M. P. Tan, Y. Huang, F. G. Omenetto, J. A. Rogers, and M. R. Bruchas: Science 340 (2013) 211. https://doi.org/10.1126/science.1232437

7 J. G. McCall, T.-I. Kim, G. Shin, X. Huang, Y. H. Jung, R. Al-Hasani, F. G. Omenetto, M. R. Bruchas, and J. A. Rogers: Nat. Protoc. 8 (2013) 2413. https://doi.org/10.1038/nprot.2013.158

8 G. Shin, A. M. Gomez, R. Al-Hasani, Y. R. Jeong, J. Kim, Z. Xie, A. Banks, S. M. Lee, S. Y. Han, C. J. Yoo, J.-L. Lee, S. H. Lee, J. Kurniawan, J. Tureb, Z. Guo, J. Yoon, S.-I. Park, S. Y. Bang, Y. Nam, M. C. Walicki, V. K. Samineni, A. D. Mickle, K. Lee, S. Y. Heo, J. G. McCall, T. Pan, L. Wang, X. Feng, T.-I. Kim, J. K. Kim, Y. Li, Y. Huang, R. W. Gereau IV, J. S. Ha, M. R. Bruchas, and J. A. Rogers: Neuron 93 (2017) 509. https://doi. org/10.1016/j.neuron.2016.12.031 
9 S. T. Lee, P. A. Williams, C. E. Braine, D. Lin, S. W. M. John, and P. P. Irazoqui: IEEE Trans. Neural Syst. Rehabil. Eng. 23 (2015) 655. https://doi.org/10.1109/TNSRE.2015.2391282

10 S. I. Park, D. S. Brenner, G. Shin, C. D. Morgan, B. A. Copits, H. U. Chung, M. Y. Pullen, K. N. Noh, S. Davidson, S. J. Oh, J. Yoon, K.-I. Jang, V. K. Samineni, M. Norman, J. G. Grajales-Reyes, S. K. Vogt, S. S. Sundaram, K. M. Wilson, J. S. Ha, R. Xu, T. Pan, T.-i. Kim, Y. Huang, M. C. Montana, J. P. Golden, M. R. Bruchas, R. W. Gereau IV, and J. A. Rogers: Nat. Biotechnol. 33 (2015) 1280. https://doi.org/10.1038/nbt.3415

11 D. Brunelli, C. Moser, L. Thiele, and L. Benini: IEEE Trans. Circuits Syst. I Regul. Pap. 56 (2009) 2519. https://doi.org/10.1109/TCSI.2009.2015690

12 H. Kim, Y. Min, C. Jeong, K.-Y. Kim, C. Kim, and S. Kim: IEEE Trans. Circuits Syst. II Exp. Briefs 60 (2013) 331. https://doi.org/10.1109/TCSII.2013.2258262

13 J. Sankman, H. Chen, and D. Ma: Proc. 2011 IEEE Int. Symp. Circuits Systems (IEEE, 2011) 2541. https://doi. org/10.1109/ISCAS.2011.5938122

14 W. Nattakarn, M. Haruta, T. Noda, K. Sasagawa, T. Tokuda, M. Sawan, and J. Ohta: 39th Annu. Int. Conf. IEEE Engineering in Medicine and Biology Society (EMBC'17) (2017) FrDT16-05.1.

15 W. Nattakarn, M. Haruta, T. Noda, K. Sasagawa, T. Tokuda, M. Sawan, and J. Ohta: Jpn. J. Appl. Phys. 57 (2018) 04FM05. https://doi.org/10.7567/JJAP.57.04FM05

16 A. S. Weddell, G. V. Merrett, and B. M. Al-Hashimi: IEEE Trans. Circuits Syst. I Regul. Pap. 59 (2012) 1196. https://doi.org/10.1109/TCSI.2011.2173393

17 S. Mondal and R. P. Paily: 19th Int. Symp. VLSI Design and Test (2015) 1. https://doi.org/10.1109/ ISVDAT.2015.7208108

18 T. Tokuda, T. Ishizu, W. Nattakarn, M. Haruta, T. Noda, K. Sasagawa, M. Sawan, and J. Ohta: AIP advances 8 (2018) 045018. https://doi.org/10.1063/1.5024243 